\section{EXPERIENCE WITH REPAIR OF CONGENITAL HEART DEFECTS USING ADJUNCTIVE ENDOVASCULAR DEVICES}

The use of endovascular devices as an adjunct to repair of congenital heart anomalies represents a novel but unproven therapeutic approach. Intraoperative implantation of pulmonary arterial stents (5 to $15 \mathrm{~mm}$ diameter) was done in 11 patients with pulmonary atresia with ventricular septal defect $(n=$ 4), classic tetralogy of Fallot $(n=2)$, truncus arteriosus $(n=1)$, hypoplastic left heart syndrome (stage II $[n=1]$ and stage III [n=1] Norwood procedure), and miscellaneous pulmonary arterial stenoses $(n=3)$, as well as in patients with congenital $(n=1)$ and postoperative $(n=3)$ pulmonary venous osbstruction and in 1 patient with combined pulmonary arterial and venous obstruction. The stents were effective at achieving immediate patency in all patients. There were two early deaths, one related to acute thrombosis of a small-diameter left pulmonary artery stent. Reintervention because of stentrelated pulmonary arterial stenosis was frequently necessary. In five of seven patients who survived more than 1 month after implantation of stent size $8 \mathrm{~mm}$ or smaller severe stent-related pulmonary arterial obstruction developed. In four of the five patients with pulmonary vein stent implantation intractable obstruction developed, resulting in death in all three patients who had bilateral pulmonary vein stent implantation. Intraoperative occlusion of apical muscular ventricular septal defect with use of a clamshell device inserted from the right atrial approach was accomplished in four patients. One patient who underwent associated aortic arch reconstruction died as a result of left ventricular hypoplasia. The results in the remaining three patients were favorable on the basis of absence of significant late residual intraventricular shunting, left ventricular dysfunction, or arrhythmia. We conclude that recurrent intraluminal obstruction as a result of neointimal hyperplasia appears to be an eventual certainty in currently designed small-diameter endovascular stents. For this reason, we would recommend standard surgical techniques for repair of obstructive lesions of the pulmonary arterial confluence to maximize growth potential. Device occlusion of muscular ventricular septal defects is feasible but probably only indicated for complex cases of ventricular septal deficiency that otherwise necessitate a left ventriculotomy. (J Thorac Cardiovasc Surg 1995;110:1513-20)

John G. Coles, MD, ${ }^{a}$ Ilya Yemets, MD $^{\text {a }}$ (by invitation), Hani K. Najm, MD (by invitation), Jeanne M. Lukanich, $\mathrm{MD}^{\mathrm{a}}$ (by invitation), Jean Perron, $\mathrm{MD}^{\mathrm{a}}$ (by invitation), Greg J. Wilson, $\mathrm{MD}^{\mathrm{c}}$ (by invitation), Marlene Rabinovitch, $\mathrm{MD}^{\mathrm{b}}$ (by invitation), David G. Nykanen, $\mathrm{MD}^{\mathrm{b}}$ (by invitation), Lee N. Benson, $\mathrm{MD}^{\mathrm{b}}$ (by invitation), Ivan M. Rebeyka, $\mathrm{MD}^{\mathrm{a}}$ (by invitation), George A. Trusler, MD, ${ }^{\mathrm{a}}$ Robert M. Freedom, MD, ${ }^{\mathrm{b}}$ and William G. Williams, MD, ${ }^{\mathrm{a}}$

Toronto, Ontario, Canada
O perative repair of congenital cardiac lesions provides access for the deployment of endovascular stents as a competitive option to conventional patch

From the Divisions of Cardiovascular Surgery, ${ }^{\mathrm{a}}$ Cardiology, ${ }^{\mathrm{b}}$ and Pathology, ${ }^{c}$ The Hospital for Sick Children, Toronto, Ontario, Canada

Read at the Seventy-fifth Annual Meeting of The American Association forr Thoracic Surgery, Boston, Mass., April 23-26, 1995. angioplasty techniques in the management of congenital and acquired obstruction within the pulmonary arterial and venous systems. Although the early

Address for reprints: John G. Coles, MD, The Hospital for Sick Children, 555 University Ave,, Toronto, Ontario, Canada M5G 1 X8.

Copyright (C) 1995 by Mosby-Year Book, Inc.

$0022-5223 / 95 \$ 5.00+0 \quad \mathbf{1 2 / 6 / 6 7 3 8 4}$ 
accrued experience with percutaneous transcatheter insertion of endovascular stents for balloon dilationresistant pulmonary arterial stenosis has been favorable ${ }^{1-3}$ there is limited information concerning the intermediate-term effects of these devices on the pulmonary vasculature.

Preoperative transcatheter closure of congenital muscular ventricular septal defects (VSDs) has been reported to facilitate subsequent surgical repair of associated lesions and, in some cases, to obviate the need for a left ventricular incision for patch closure of complex deficiency of the ventricular septum. ${ }^{4}$ Intraoperative insertion of clamshell devices has been successfully used to occlude muscular VSDs and may be accomplished with greater technical simplicity than can be achieved with the use of interventional catheterization techniques. ${ }^{5}$

Review of our experience with adjunctive endovascular devices with intermediate-term follow-up revealed several important problems that seriously detract from the apparent advantage of technical simplicity in comparison with conventional surgical techniques. The major problem identified with the endovascular stents is the virtually invariable development of recurrent intraluminal obstruction, which seriously complicated the treatment of patients who received small-diameter stents for treatment of pulmonary arterial and venous obstructive lesions.

\section{Patients and methods}

The complete experience with intraoperative implantation of endovascular devices at The Hospital for Sick Children, Toronto, includes 20 patients who underwent the following procedures during the period October 1991 to August 1994: stenting of branch pulmonary arteries $(n=11)$, stenting of pulmonary veins $(n=4)$, combined pulmonary arterial and venous implantation $(n=1)$, and device closure of muscular VSD $(n=4)$.

Pulmonary artery stents. The morphologic diagnoses of the 11 patients who underwent pulmonary arterial stent implantation (Palmaz stainless steel intraluminal stent, Johnson and Johnson Systems, Somerville, N.J.) included pulmonary atresia with VSD $(n=4)$, classic tetralogy of Fallot $(n=2)$, truncus arteriosus $(n=1)$, hypoplastic left heart syndrome (stage II $[n=1]$ and stage III $[n=1]$ Norwood procedure), and ( $n=1$ each) aortopulmonary window, pulmonary atresia with intact ventricular septum, and tricuspid atresia with absent pulmonary valve syndrome. One patient with tetralogy of Fallot underwent simultaneous stent implantation into the right pulmonary artery and the left pulmonary veins.

The patients ages ranged from 4 days to 16 years. All patients have been evaluated with postoperative cardiac catheterization and angiography, and the mean follow-up period is 24.7 months. Pulmonary arterial stents were implanted unilaterally in seven patients and bilaterally in five. Intraoperative stent implantation was done at the time of primary repair in five patients and at a reoperation in eight patients.

Pulmonary venous stent implantation. Pulmonary venous stent implantation was done in five patients. In three patients pulmonary vein stenosis developed subsequent to repair of supracardiac $(n=1)$ or coronary sinus $(n=2)$ total anomalous pulmonary venous connection; the remaining patients had presumably congenital pulmonary vein stenosis in association with pulmonary atresia with intact ventricular septum $(n=1)$ and tetralogy of Fallot $(n=1)$. Bilateral pulmonary venous stent implantation was done in four patients and unilateral pulmonary venous stenting, to both the upper and lower lobe branches, in two patients. The stent diameter ranged from 6 to 10 $\mathrm{mm}$ at the time of implantation.

Technique of endovascular device insertion. Intraoperative stent implantation was done under direct vision over a balloon dilation catheter to achieve an effective stent diameter ranging from 5 to $15 \mathrm{~mm}$. In all cases, the stents were inserted across the ostium of one or both pulmonary arteries such that a short segment of the expanded stent remained within the main pulmonary trunk. In the case of obstruction involving the ostia of pulmonary veins, a short length of the stent protruded into the left atrium proper.

Device occlusion of muscular VSD. Intraoperative device occlusion of a muscular VSD was done in three neonates and one infant (weight range 3.0 to $8.1 \mathrm{~kg}$ ). The position of the defect was apical in three patients and midmuscular in one.

VSD closure was accomplished with a $17 \mathrm{~mm}$ USCI clamshell device (Bard Clamshell Septal Umbrella, USCI Division, C.R. Bard, Billerica, Mass.) in three patients; a Rashkind ductal occlusion device was used in one patient. Operation was done for the express purpose of device closure of a muscular VSD in two patients; 1 patient each underwent concomitant patch enlargement of branch pulmonary arteries and polytetrafluoroethylene patch angioplasty of a hypoplastic aortic arch.

Technique used for device occlusion. The muscular VSD was identified by probing the left ventricular aspect of the septum with a right-angled device passed through an associated atrial septal defect from the right atrial approach. The right-angled clamp was then used to grasp a large silk suture attached to the clamshell device delivery sheath. The silk suture was then used to guide the delivery sheath across the VSD through the right ventricular orifice, facilitating sequential release and engagement of the left ventricular and right ventricular components of the clamshell device. The device was directly inserted into the single patient with a midmuscular VSD without suture guidance.

\section{Results}

Pulmonary arterial stent implantation. There were three deaths in this group. One patient died with low cardiac output syndrome after bilateral pulmonary arterial stent implantation to correct pulmonary arterial confluence stenosis after repair of truncus arteriosus. One patient with tricuspid 
atresia and absent pulmonary valve syndrome with a left pulmonary artery stent died as a result of sepsis and inability to regulate pulmonary blood flow. The third patient died after bilateral pulmonary arterial stent implantation done at the time of repair of pulmonary atresia with VSD (described later).

Reinterventions for stent-related pulmonary arterial stemosis. Ten eligible patients underwent 13 postoperative cardiac catheterizations at a mean interval of 9.6 months after stent implantation. On the basis of results of the first cardiac catheterization, there was a mean reduction of $56 \%$ in the effective caliber of the stented pulmonary artery relative to intraoperative measurements. Dilation of the stented pulmonary artery segments was done in 6 of 10 eligible patients and in 6 of 13 eligible stented branch pulmonary arteries; 3 patients required 2 dilation procedures and 1 patient required 3. Variable improvement in the caliber of stented pulmonary artery segments on the basis of angiographic assessment was observed after stent dilation.

Five of seven patients who survived more than 1 . month after implantation of a stent size $8 \mathrm{~mm}$ or smaller had the development of severe stent-related pulmonary arterial obstruction.

Reoperation to correct stent-related stenosis of the pulmonary arterial confluence was successfully done in two patients by pericardial patch arterioplasty at 8 and 12 months after initial stent implantation.

Neonatal pulmonary atresia with VSD. Three patients underwent stent implantation in an attempt to overcome ductal-related constriction in the left pulmonary artery at the time of neonatal repair. In one patient, this necessitated implantation of two overlapping stents in the left pulmonary artery to effectively straddle the area of ductal insertion. In this patient thrombosis developed within the stents resulting in a $1 \mathrm{~mm}$ residual patent lumen, which led to death 2 weeks after operation. In all three patients, $5 \mathrm{~mm}$ stent implantation into the ostium of the left pulmonary artery resulted in distortion of the ostium of the contralateral right pulmonary artery, which necessitated right pulmonary artery stent implantation concomitantly $(n=2)$ or at a reoperation $(n=1)$. Reoperation for stent-related pulmonary arterial stenosis (bilateral in 1; unilateral in 1) was required at 4 months and 12 months, respectively, after initial stent implantation (Fig. 1). In both cases, the stents were opened lengthwise and the branch pulmonary arteries successfully augmented with pericardial patches.
Pulmonary venous stent implantation. The three patients with bilateral stent implantation died at 4 , 15 , and 24 months after operative stent implantation as a result of progressive, bilateral, intraluminal obstruction. The obstruction occurred within the stented segment in all patients and extended into nonstented parenchymal segments of the pulmonary veins bilaterally in one patient (Fig. 2). Among these three nonsurviving patients, one underwent an attempted stent dilation and another an unsuccessful revision with excision of neointimal hyperplasia. Among the two surviving patients who had unilateral pulmonary venous stent implantation, one underwent balloon dilation with modest caliber improvement at 17 months after implantation; the other patient is doing well clinically with no evidence of obstruction in the single pulmonary vein branch evaluable by two-dimensional echocardiography.

Device closure of muscular VSD. The patient with a midmuscular VSD died with low cardiac output syndrome. Postmortem examination demonstrated satisfactory occlusive position of the device; the presence of moderately severe left ventricular and mitral annular hypoplasia was the presumed cause of death (Fig. 3). One patient in whom the Rashkind ductal occlusion device was used required reoperation to close a peridevice residual defect in association with a residual leak of a perimembranous defect originally treated by patch closure. This patient still had a small residual VSD at the time of most recent follow-up. In one patient unexplained complete heart block developed subsequent to device closure of an apical muscular VSD.

In the two surviving patients treated with a clamshell occlusion device followed up 11 to 52 months after implantation, there is no evidence of residual intraventricular shunting or ventricular dysfunction as assessed by postoperative echocardiography and left ventricular angiography.

\section{Discussion}

Transcatheter deployment of balloon-expandable endovascular stents has been successfully applied in the treatment of congenital and acquired pulmonary arterial stenosis. ${ }^{1,2}$ This technique has also been successfully used to increase the caliber of aortopulmonary collaterals, ${ }^{6}$ obstructed right ventricular outflow tract conduits, ${ }^{7}$ and systemic and pulmonary venous pathways after the atrial switch operation ${ }^{8}$ and as a means to maintain patency of the ductus arteriosus $^{6}$ and the native right ventricular outflow 

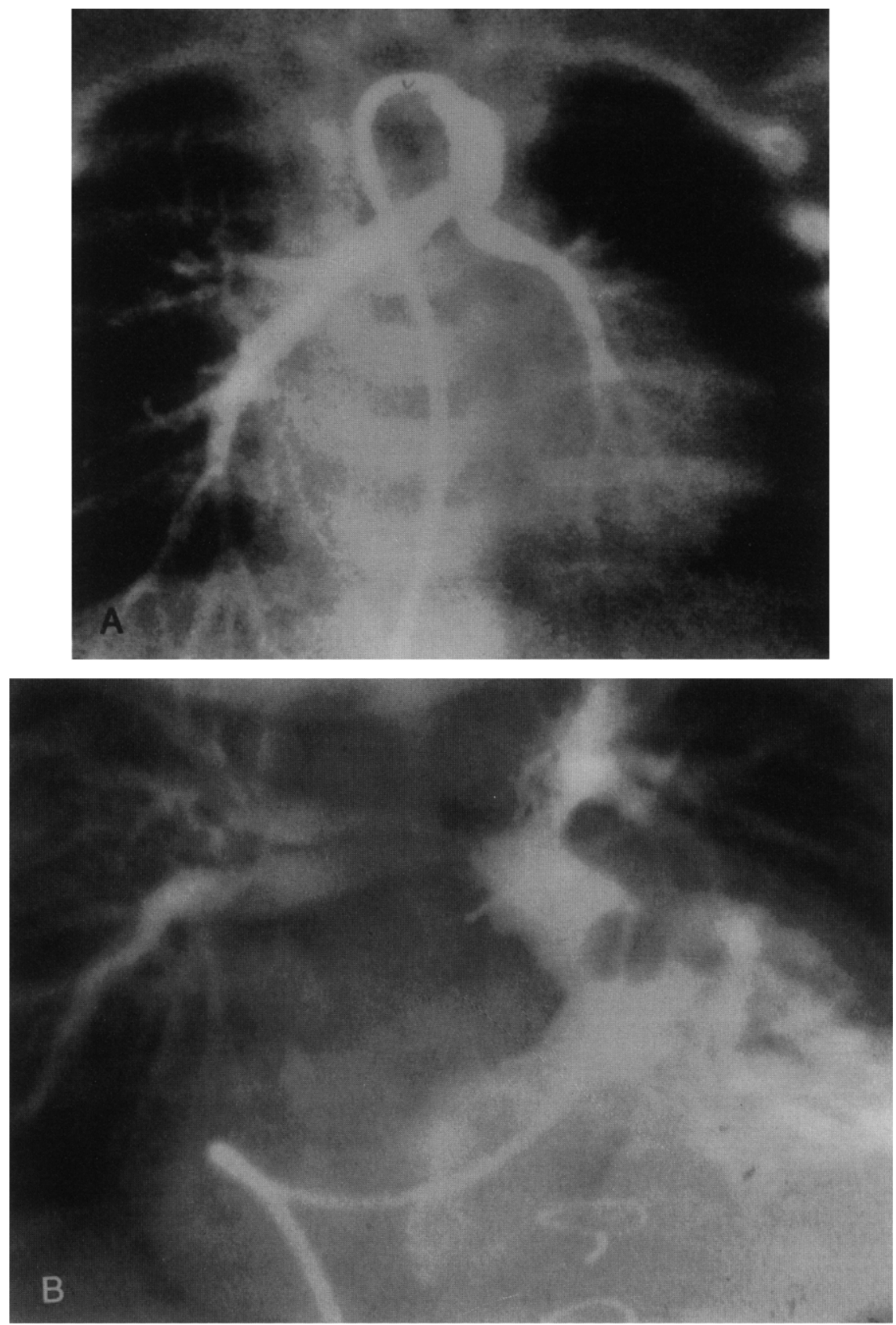

Fig. 1. A, Preoperative angiogram in neonate with pulmonary atresia and VSD showing ductal insertion onto origin of left pulmonary artery producing potential for pulmonary arterial confluence stenosis. $\mathbf{B}$, Severe, bilateral proximal pulmonary artery stenosis that occurred four months after insertion of endovascular stents across origin of both branch pulmonary arteries and inflation of stents to $5 \mathrm{~mm}$ diameter.

tract in pulmonary atresia after radiofrequency perforation. ${ }^{9,} 10$ Mendelsohn and colleagues ${ }^{5}$ first reported the use of intraoperative endovascular stents, citing the potential advantages of this approach in patients undergoing the Fontan procedure in whom percutaneous vascular access to the pulmonary arteries may be difficult preoperatively.

Review of our experience with intraoperative balloon-expandable stent implantation revealed a high prevalence of recurrent intraluminal obstruc- 


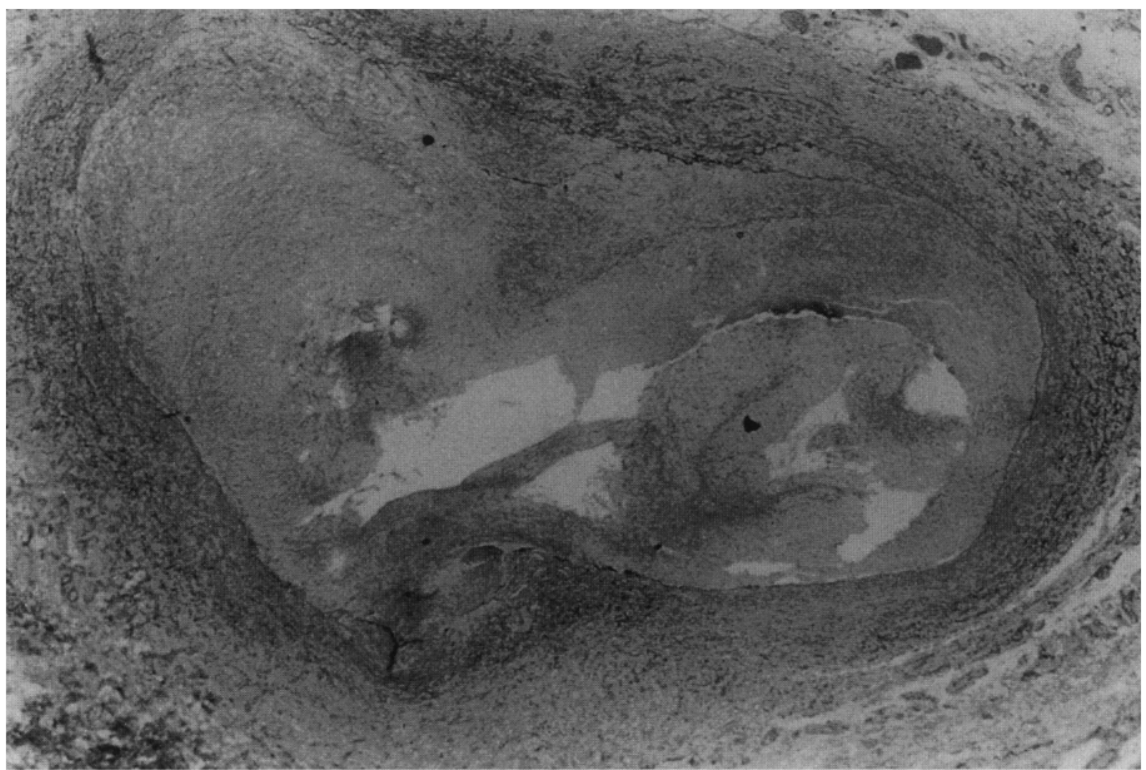

Fig. 2. Section taken through right upper lobe pulmonary vein immediately upstream of site of endovascular stent implantation. Severe intimal hyperplasia is present, attributable to smooth muscle cell proliferation and extensive extracellular matrix material, consisting of predominantly glycosaminoglycans, collagen, and disorganized elastic fiber production (Movat stain; original magnification $\times 20$ ).

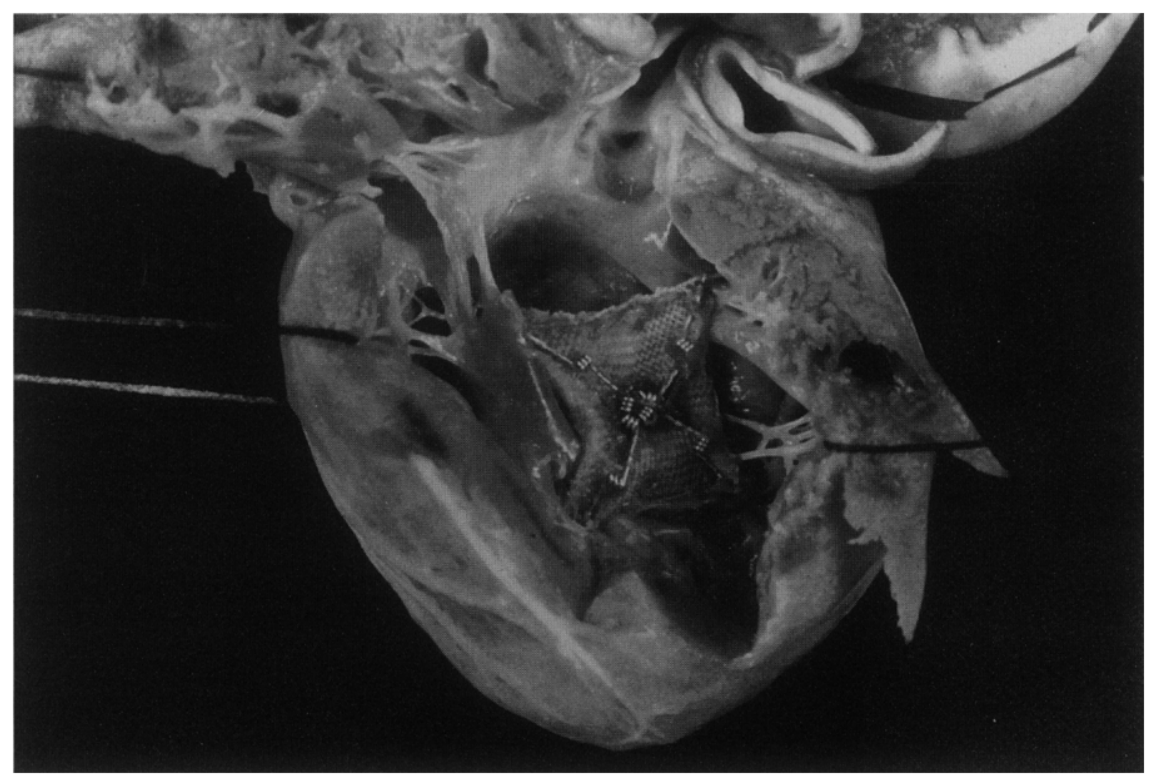

Fig. 3. Autopsy specimen illustrating satisfactory left ventricular position of $17 \mathrm{~mm}$ USCI clamshell device. Marked mitral annular and left ventricular hypoplasia were present.

tion within the stent. This finding was especially conspicuous and hemodynamically important in small-diameter stents placed in the neonatal pulmonary arterial confluence, but was also observed in larger stents up to $16 \mathrm{~mm}$ in initial implant diameter.

This result is consistent with our institutional experience with percutaneous transcatheter inser- 
tion of balloon-expandable endovascular stents. Follow-up cardiac catheterization and selective pulmonary artery angiography done in 42 patients who underwent this procedure identified endoluminal tissue ingrowth occurring to varying degrees in all stents; subsequent further dilation was required in 11 patients (26\%) during a median follow-up of 15 months. ${ }^{3}$ Usually a modest but significant improvement in the caliber of the vessel at the site of the stent was accomplished by dilation both in the present surgical series and in the group undergoing transcatheter pulmonary arterial stent implantation. In contrast, O'Laughlin and colleagues ${ }^{2}$ reported angiographic evidence of restenosis in only 1 of 25 patients undergoing transcatheter pulmonary arterial stent implantation, although in the majority of stents there was a 1 to $2 \mathrm{~mm}$ thick layer of intimal proliferation evident. In that series, redilation of 17 stents in 14 patients increased the narrowest stent diameter from $9.0 \mathrm{~mm}$ to $11.8 \mathrm{~mm}(p<0.01$ ), implying a consistent but finite susceptibility to increasing the effective diameter of chronically implanted stents. The higher rate of stent-related obstruction in our institutional experience with interventional and surgical stent implantation relative to that of O'Laughlin and colleagues ${ }^{2}$ is likely related to the greater size and age range and the predominant use of larger "iliac" stents in their series.

The development of stent-related obstruction was especially pernicious in the group of patients who underwent stent implantation to correct pulmonary venous obstruction. All five patients who underwent operative stent implantation to correct pulmonary vein stenosis, as well as two additional patients in whom a pulmonary venous stent implantation was accomplished at cardiac catheterization, had the development of progressive and intractable obstruction within months after stent implantation. This process was fatal in the three patients with bilateral pulmonary venous stent implantation. This result is consistent with that in the report from the University of Michigan Medical Center involving three patients who had stent implantation to correct pulmonary vein stenosis. ${ }^{5}$

Restenosis occurring within endovascular stents appears to be a consequence of luminal encroachment by neointimal hyperplasia. ${ }^{11}$ The initial tissue response after stent implantation is that of thrombus deposition in proximity to the stents with retention of intervening endothelial cells. ${ }^{12}$ Within a few days to weeks, smooth muscle cell proliferation, neovas- cularization, and extracellular matrix deposition develop eccentrically from the site of the stent latticework. The precise stimuli that cause cellular proliferation and synthesis of intracellular matrix in the presence of metallic stents, as well as the potential for remodeling and regression, remain unknown. ${ }^{13,14}$ The tendency for the development of late restenosis is well recognized after coronary arterial stent implantation, which occurs with a time course and frequency similar to that after balloon angioplasty. ${ }^{15,16}$ Both in the coronary artery experience $^{17}$ and in our own experience with percutaneous transcatheter pulmonary arterial stent implantation, ${ }^{3}$ stent oversizing has been identified as a risk factor for restenosis and apparently leads to obstructive lesions at the junction between the stent and the native vessel in cases in which the stent size is significantly larger than the adjacent vessel.

Additional considerations militate against the use of small-diameter endovascular stents in the management of pulmonary arterial confluence stenosis in neonates and infants. In our experience, stent implantation across the origin of the left pulmonary artery to treat ductus-related stenosis invariably compromised the origin of the contralateral right pulmonary artery. Moreover, implantation of endovascular stents into growing pulmonary arteries may have the effect of fixing the caliber of the vessel and impairing the growth potential. Our experience suggests that further dilation of chronically implanted endovascular stents is usually possible, but limited in extent and unlikely to achieve maximal dilation to the nominal balloon-expandable stent diameter.

On the basis of these considerations, we would consider conventional patch angioplasty techniques preferable to the use of small-diameter endovascular stents in the treatment of pulmonary arterial and pulmonary venous obstruction, recognizing the poor prognosis associated with the diffuse forms of pulmonary venous obstruction with any treatment modality. On the other hand, percutaneous transcatheter deployment of endovascular stents may represent the safest and most effective method to achieve satisfactory vessel caliber in obstructed pulmonary arteries of any size in the immediate postoperative period, in which circumstances surgical revision may carry an unacceptably high risk. Improvements in coronary artery stent design, such as the development of drug-eluting and biodegradable polymer stents, may ultimately be beneficially applicable to obstructive congenital lesions. ${ }^{15}$

Our small experience with percutaneous trans- 
catheter and intraoperative device occlusion of muscular VSDs indicates that these approaches are effective in the short-term in providing closure of the defect. This finding supports those of Bridges and associates ${ }^{4}$ and Fischberger, Bridges, and Keane, ${ }^{18}$ who additionally concluded that, in the case of multiple VSDs, transcatheter closure was preferable to intraoperative closure inasmuch as it allows angiographic definition and immediate management of residual defects after initial device implantation. The device-related perforation of the left ventricle in one of our patients, however, raises concerns about the long-term durability and safety of ventricular septal devices. With this in mind, and on the basis of our favorable experience with conventional patch closure of apical muscular VSDs by use of a limited apical right ventricular incision, we would currently reserve consideration of device closure of VSDs for complex cases of severe ventricular septal deficiency that otherwise necessitate a left ventriculotomy.

\section{REFERENCES}

1. O'Laughlin MP, Perry SB, Lock JE, Mullins CE. Use of endovascular stents in congenital heart disease. Circulation 1991;83:1923-39.

2. O'Laughlin MP, Slack MC, Grifka RG, Perry SB, Lock JE, Mullins CD. Implantation and intermediateterm follow-up of stents in congenital heart disease. Circulation 1993;88:605-14.

3. Fogleman R, Nykanen D, Smallhorn JF, McCrindle BW, Freedom RM, Benson LN. Endovascular stents in the pulmonary circulation: clinical impact on management and medium-term follow-up. Circulation (In press).

4. Bridges ND, Perry SB, Keane JF, et al. Preoperative transcatheter closure of congenital muscular ventricular septal defects. N Engl J Med 1991;324:1312-7.

5. Mendelsohn AM, Bove EL, Lupinetti FM, et al. Intraoperative and percutaneous stenting of congenital pulmonary artery and vein stenosis. Circulation 1993;88:II210-7.

6. Zahn EM, Lima VC, Benson LN, Freedom RM. Use of endovascular stents to increase pulmonary blood flow in pulmonary atresia with ventricular septal defect. Am J Cardiol 1992;70:411-2.

7. Hosking MC, Benson LN, Nakanishi T, Burrows PE, Williams WG, Freedom RM. Intravascular stent prosthesis for right ventricular outflow obstruction. J Am Coll Cardiol 1992;20:373-80.

8. Chatelain P, Meier B, Friedli B. Stenting of the superior vena cava and inferior vena cava for symptomatic narrowing after repeated atrial surgery for transposition of the great vessels. Br Heart J 1991;66: 466A.

9. Gibbs JL, Roffman MT, Rees MR, Parson JM, Blackburn ME, Ruiz CE. Stenting the arterial duct: a new approach to palliation for pulmonary atresia. $\mathrm{Br}$ Heart J 1992;67:240-5.

10. Hausdorf G, Schneider M, Lange P. Catheter creation of an open outflow tract in previously atretic right ventricular outflow tract associated with ventricular septal defect. Am J Cardiol 1993;72:354-6.

11. Gordon PC, Gibson M, Cohen DJ, Carrozza JP, Kuntz RE, Baim DS. Mechanisms of restenosis and redilation within coronary stents: quantitative angiographic assessment. J Am Coll Cardiol 1993;21:116674.

12. Palmaz JC. Intravascular stents: tissue-stent interactions and design. AJR Am J Roentgenol 1993;160: 613-8.

13. Ruobin GS, King SB, Douglas JS, Lembo NJ, Robinson KA. Intracoronary stenting during percutaneous transluminal coronary angioplasty. Circulation 1990; 81(Suppl):IV92-100.

14. White IW, Ramee SR, Banks AK, et al. A new balloon-expandable tantalum coral stent: angiographic patency and histologic findings in an athrologenic swine model. J Am Coll Cardiol 1992;19:870-6.

15. De Jaegere P, De Feyter PJ, van der Giessen WJ, et al. Endovascular stents: preliminary clinical results and future developments. Clin Cardiol 1993;16:36978.

16. Kastrati A, Schomig A, Dietz R, Newmann FJ, Richardt $\mathrm{G}$. Time course of restenosis during the first year after emergency coronary stenting. Circulation 1993; 87:1498-505.

17. Haude M, Erbel R, Straub U, et al. Short- and long-term results after intracoronary stenting in human coronary arteries: monocenter experience with the balloon-expandable Palmaz-Schatz stent. $\mathrm{Br}$ Heart J 1991;66:337-45.

18. Fischberger SB, Bridges ND, Keane JF. Intraoperative device closure of ventricular septal defect. Circulation 1993;88:II205-9.

\section{Discussion}

Dr. Pedro J. del Nido (Boston, Mass.). The experience at Boston Children's Hospital with device closure of VSDs, which has been previously reported, includes a total of 10 patients. Four patients had multiple VSDs, or "Swiss cheese septum," and eight had additional complex defects. The overall results included three early deaths, two of which were related to residual shunts that were significant and compromised cardiac output. The third was related, at least in part, to the manipulation required to insert this occluder device in the apex. This was a result of difficult visibility and retraction of the ventricle.

One additional problem that may come up, and we would like to ask the authors if they have seen this, is the 
inability to determine where the distal end of the balloon is in relation to the branch pulmonary arteries. One of the potential problems is inserting the guide wire into a small branch, causing a tear or an aneurysmal dilatation of the branch pulmonary arteries when the balloon is dilated. Although the authors stated that all the balloons were inserted under direct vision, the distal end of the balloon cannot be visualized by this modality.

The problems that we have found with intraoperative interventions, in summary, are, first, with VSD occlusions, the inability to detect residual defects or residual obstruction before discontinuation of bypass; second, the inability to determine the distal position of the dilating balloon or of the stent, which may lead to pulmonary artery damage distally; and, finally, the difficulty in exposure that occurs when one is attempting to close the VSD. This is particularly true with apical VSDs.

One additional question that I would like to ask is whether the authors noticed in their experience any differences between the apical and the midmuscular VSDS with respect to the ease of insertion of the device and with the degree of distortion required for exposure. Could, perhaps, the small but significant prevalence of heart block reported be related to the manipulation of the ventricle?

Dr. Coles. We have no experience in using these devices in cases of either percutaneously or intraoperatively, and agree that it may be advantageous to do such procedures in the catheterization laboratory so that one can identify any residual leaks, because it has been shown that many of these patients with complex left ventricular VSD, require insertion of multiple devices to successfully achieve occlusion.

I also concur that positioning of stents in the distal pulmonary artery tree intraoperatively may be difficult, simply because, as indicated, one cannot determine securely where the distal extent of the stent is. In our series the stents were placed across the origin of the bronchopulmonary artery, so it was less of a problem. However, in one baby, weary of trying to basically obviate a ductal constriction-related stenosis, we finally used two stents, and I think our inability to adequately position the distal end of the more distal stent contributed to the acute thrombosis in that patient.

In terms of exposure of the apical VSDs, we found that the technique was fairly straightforward, although I agree that the unexplained complete heart block in one patient might be related to manipulation.

With respect to your question about apical defects, we have experience closing several of these defects with use of an apical right ventricular incision rather than an apical left ventricular incision, and we think that is the preferred way to do it, particularly in view of our experience in the one patient in whom the device migrated. We also heard recently from Dr. Lock that there is a fairly high prevalence of strut fracture, not only in the atrial position but also in the ventricular position, so we think it is safer and more secure to close these lesions by a convention patch closure, working through either the right atrium or the right ventricle.

Dr. Richard A. Hopkins (Washington, D.C.). I have two questions. First, I am going to pin you down as to your feeling about what size stent is too small: what is your cutoff for using stents in the pulmonary arteries now? Second, in the patients who underwent reoperation after having stents placed, did you remove the stent or did you cut across it, and how difficult was that operation?

Dr. Coles. There were seven patients, I believe, who had a stent size of $8 \mathrm{~mm}$ or less, at least that was the diameter that they were inflated to at the time of the initial implant, and five of those patients had severe recurrent obstruction necessitating intervention either in the form of operation or one or more dilations. Having said that, I do not think that is necessarily a discrete threshold size, because there were patients with larger stents, including a patient with a 15 or $16 \mathrm{~mm}$ stent, who required further dilations. I do not think any stent size is immune from the problem of neointimal hyperplasia.

There were two patients in this series in whom we had to cut across the stent, plus some additional patients who underwent percutaneous transcatheter insertion of endovascular stents, who subsequently required a pulmonary arterioplasty. This can be done, but it is not easy, because the marginal protruding stents must be removed to secure the patch in position. When that is done and when they are peeled back, they tend to be well heeled into the pulmonary artery, and thus the pulmonary artery can become frayed, making the subsequent suturing difficult, although we were able to do it. I think this is another disadvantage of the stents. 